\title{
Evaluation of 3D Object Manipulation on Multi-touch Surfaces Using Unconstrained Viewing Angles
}

\author{
Daniel Mendes and Alfredo Ferreira \\ INESC-ID / IST / Technical University of Lisbon, \\ Rua Alves Redol, 9, \\ 1000-029 Lisboa, Portugal \\ \{danielmendes, alfredo.ferreira\}aist.utl.pt
}

\begin{abstract}
Recently, considerable research has been carried out regarding threedimensional object manipulation on multi-touch surfaces. However, most current solutions were developed having in mind scenarios with a camera perpendicular to a scene axis, and cannot be easily used to manipulate threedimensional objects in unconstrained viewpoints. In this paper, we present and evaluate a set of object manipulation techniques. As a test bed for this study, we used an unconstrained virtual LEGO modeling tool, taking advantage of ongoing work and popularity of LEGO construction among people of all ages. From this evaluation we improved our understanding on how users prefer to manipulate 3D objects on multi-touch surfaces.
\end{abstract}

Keywords: Multi-touch interaction, 3D object manipulation, rotation, translation, user evaluation.

\section{Introduction}

From mobile devices to tabletops or wall-sized interaction surfaces, multi-touch is becoming part of our everyday life. Nowadays it is possible to build low-cost prototypes that can detect multiple simultaneous touches. This kind of technology is becoming more and more popular among the general public.

With the flourishing of these devices, new interaction paradigms are needed. Multi-touch interfaces can provide more natural and user-friendly interaction than the traditional WIMP interfaces. Indeed, studies show that multi-touch interfaces are also more efficient $[6,2]$. Although new paradigms have already been defined in some scenarios, such as the manipulation of two-dimensional objects, other scenarios are in need of more work, such as the manipulation of three-dimensional objects.

Most research in this context were designed for scenes with a camera perpendicular to one of the axis, making it difficult to apply the proposed techniques in scenarios which allows the freely manipulation of the camera and need to precisely position and orientate an object. To have such scenario and address this challenge, we used a prototype of virtual LEGO models in a multi-touch table. In this paper, we propose a set of translation and rotation techniques, adapted from existing approaches, and evaluate their suitability for unconstrained viewpoints, comparing them with each other and with existing solutions. 


\section{Related Work}

The research on object manipulation using multi-touch surfaces started with 2D objects. Hancock et al. [3] surveyed several techniques for both rotation and translation of this type of objects, using multi-touch based gestures. One of these techniques, the two-point rotation and translation, has become the de facto standard.

When manipulating of three-dimensional objects, the challenges are different. The main difference is the lack of direct mapping from input to visualization. Instead, it is necessary to map the 2D input to a 3D view. Several approaches were proposed to tackle this challenge. Hancock et al. [4] presented a study about manipulating 3D objects using one, two and three touches simultaneously. The approach using three touches showed the best results when tested and was later denoted as StickyFingers [5]. Martinet et al. [7] developed two techniques to translate 3D objects: one extends the standard four viewports that can be found in many CAD applications, and another (Z-technique) uses only a perspective view of the scene.

The work presented above, regarding interaction with $3 \mathrm{D}$ objects on multi-touch surfaces, were made having in mind scenes where the camera does not move, or at least does not rotate, since the basic movement of the objects is done in a plane parallel to the viewing plane. Moreover, scenes with a different camera, such as an isometric perspective, will require additional cognitive effort from the user to understand the plane in which the objects are moving or, in other words, what is the relation between the viewing plane and the scene referential.

\section{3D Object Manipulation on MT Surfaces}

The test bed for our study was a prototype of virtual LEGO modeling on table-sized multi-touch surfaces, presented in [8]. It allows touch-based manipulation of blocks, rotating and moving them freely, to build virtual LEGO models and uses an orbiting camera. The techniques used for 3D object manipulation are derived from approaches presented above and are described next.

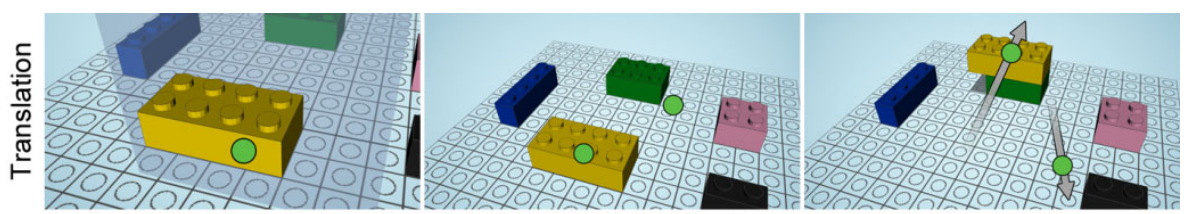

(a) Orthogonal

(b) Horizontal-Z - 1

(c) Horizontal-Z - 2

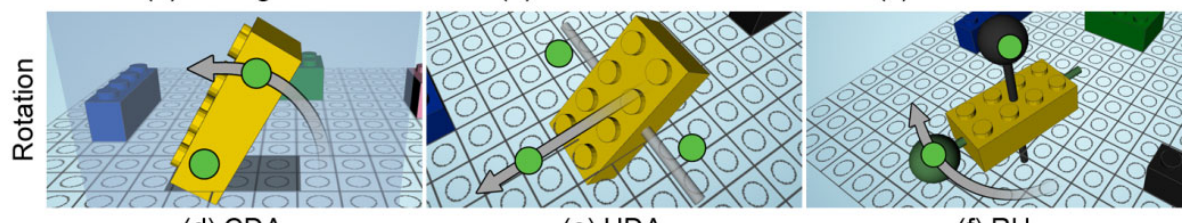

(d) CDA

(e) UDA

(f) $\mathrm{RH}$

Fig. 1. Proposed 3D object manipulation techniques for unconstrained viewpoints. The green circles represent user touches and the gray arrows the movement of these touches. 


\subsection{Translation Techniques}

Orthogonal - is based on the two-point rotation and translation for 2D objects [3]. To apply it to 3D, we use a plane orthogonal to one of the scene axes, closer to the current view vector to move the object (Fig.1.a). To use a different plane, the user will have to rotate the camera. We display a blue square representing the translation plane.

Horizontal-Z - derived from the Z-technique [7], but instead of moving the object in a plane parallel to the view, it moves the object in a horizontal plane. A second touch manipulates the depth of the object relatively to the camera (Fig.1.b,c). To improve the perception of object position, a shadow of the object being moved is cast.

Plane-Switch - uses a concept similar to the first technique. Initially, the translation is done on a horizontal plane. Touching on the blue square, the translation plane becomes the vertical plane that has the closer normal direction to the view vector. Thus, the user can move the object in $3 \mathrm{D}$ without changing the camera.

\subsection{Rotation Techniques}

Camera-Defined-Axis (CDA) - is used with the Orthogonal translation technique. While a touch is used to move the object, another touch is used to rotate the object (Fig.1.d). It uses the translation plane normal as rotation axis and the first touch as rotation center. To rotate around other axes, the user will need to rotate the camera.

User-Defined-Axis (UDA) - is similar to the Opposable Thumb [5]. The main differences are that the rotation axis is parallel to the $\mathrm{X}, \mathrm{Y}$ or $\mathrm{Z}$ axis and the rotation center is the center of the object (Fig.1.e). The rotation axis used is the one closer to that defined by the two first touches. Thus, we can use the ease to define the rotation axis introduced by the Opposable Thumb and use a rotation axis easily discernible.

Rotation-Handles (RH) - uses the concept of virtual handles [1]. When the user selects an object, three handles parallel to the scene referential become visible. One touch selects the handle corresponding to the desired rotation axis, and another touch rotates the object around its center, by dragging other handle (Fig.1.f).

\section{Comparative Evaluation}

We set out to evaluate the techniques for 3D objects manipulation described above using a virtual LEGO modeling prototype. To verify our assumptions regarding translation using a plane parallel to the view, we included the Z-technique in our evaluation. There were two different tasks, one for translations, where user had to move five bricks until their position were coincident with ones with the same color but translucent, and another for rotations, where the user had to rotate four blocks to a specific orientation. Tests involved twenty users with very distinct backgrounds and a large (greater than A0) multi-touch enabled tabletop surface.

The times taken by each user to complete the tasks with the different techniques followed a normal distribution, accordingly to the Shapiro-Wilk test. We used the One-Way ANOVA test and the Post-hoc Tukey HSD multiple comparisons test to find out significant differences in the translation. The Z-Technique was significantly 
slower than the Orthogonal and the Plane-Switch. These two approaches were also faster than the Horizontal-Z, which had a much higher rate of failure to complete the task within the available time than all approaches. Concerning the rotation, we applied the T-test to each pair of techniques and concluded that the $\mathrm{RH}$ was significantly faster than the CDA and the UDA. The RH was also the one with the lowest rate of failure. We asked users to classify each technique in a four-point Likert scale and, by applying the Wilcoxon Signed Ranks Test, we observed that their preferences reflected what was concluded with task time and rate of failure analysis.

\section{Conclusions and Future Work}

We believe that existing solutions to 3D object manipulation on multi-touch surfaces work poorly with free viewing angles. In this paper we presented techniques for manipulating objects with this kind of scenario in mind. We tested these techniques with twenty users, comparing them among each other and with the Z-technique.

From this evaluation, we were able to conclude that users perform better with approaches that translate objects accordingly to planes perpendicular to one of the scene axes, instead of a plane parallel to the view. Even though a shadow of the object was casted to improve user perception of its location, some users initially thought that the gesture for depth manipulation was scaling the object, not moving, and found difficult to perceive the actual position of the object. We also observed that, when rotating objects, few users had some notions of what axis is needed to achieve a certain rotation. Most of them tried several axes until they identified the right one to use. We will address these issues in a near future.

Despite the evaluation with a LEGO modeling tool, we believe that conclusions here presented can be applied in generic 3D modeling scenarios. Our next step is to devise a set of manipulation techniques from the results obtained in this evaluation.

\section{References}

1. Conner, B., Snibbe, S., Herndon, K., Robbins, D., Zeleznik, R., Van Dam, A.: Threedimensional widgets. In: Proc. of the 1992 Symposium on Interactive 3D Graphics (1992)

2. Fiorella, D., Sanna, A., Lamberti, F.: Multi-touch user interface evaluation for $3 \mathrm{~d}$ object manipulation on mobile devices. Journal on Multimodal User Interfaces (2009)

3. Hancock, M.S., Carpendale, S., Vernier, F.D., Wigdor, D., Shen, C.: Rotation and translation mechanisms for tabletop interaction. In: Proc. Tabletop (2006)

4. Hancock, M., Carpendale, S., Cockburn, A.: Shallow-depth 3d interaction: design and evaluation of one-, two-and three-touch techniques. In: Proc. CHI (2007)

5. Hancock, M., ten Cate, T., Carpendale, S.: Sticky tools: Full 6dof force-based interaction for multi-touch tables. In: Proc. ITS (2009)

6. Kin, K., Agrawala, M., DeRose, T.: Determining the benefits of direct-touch, bimanual, and multifinger input on a multitouch workstation. In: Proc. GI 2009 (2009)

7. Martinet, A., Casiez, G., Grisoni, L.: The design and evaluation of 3d positioning techniques for multi-touch displays. In. In: IEEE Symposium on 3D User Interfaces (2010)

8. Mendes, D., Ferreira, A.: Virtual lego modelling on multi-touch surfaces. In: WSCG 2011 Full Papers Proceedings (2011) 